\title{
Galois Connections between Semimodules and Applications in Data Mining
}

\author{
Francisco J. Valverde-Albacete and Carmen Peláez-Moreno * \\ Dpto. de Teoría de la Señal y de las Comunicaciones. \\ Universidad Carlos III de Madrid \\ Avda. de la Universidad, 30. Leganés 28911. Spain \\ fva, carmen@tsc.uc3m.es
}

\begin{abstract}
In [1] a generalisation of Formal Concept Analysis was introduced with data mining applications in mind, $\mathcal{K}$-Formal Concept Analysis, where incidences take values in certain kinds of semirings, instead of the standard Boolean carrier set. A fundamental result was missing there, namely the second half of the equivalent of the main theorem of Formal Concept Analysis. In this continuation we introduce the structural lattice of such generalised contexts, providing a limited equivalent to the main theorem of $\mathcal{K}$-Formal Concept Analysis which allows to interpret the standard version as a privileged case in yet another direction. We motivate our results by providing instances of their use to analyse the confusion matrices of multiple-input multiple-output classifiers.
\end{abstract}

\section{Motivation: the Exploration of Confusion Matrices with $\mathcal{K}$-Formal Concept Analysis}

In pattern recognition tasks, when a classifier is provided training data in the form of feature vectors tagged with an input pattern set and produces for each vector a tag within an output pattern set, the performance of the classifier can be gleaned from the collection of pairs $\left(g_{i}, m_{j}\right)$ of one input tag, $g_{i}$, for the input data and one output tag, $m_{j}$, produced by the classifier. These results are aggregated into a confusion matrix, $T$, whose element $T_{i j}$ gives a "measure" of the joint event $\left(G=g_{i}, M=m_{j}\right)$, "providing an input pattern $g_{i}$ to the classifier who then produces an output pattern $m_{j}$ ".

In the pattern recognition community we often encounter methods that use confusion matrices to analyse classification results. However, most of the times the analysis is manual and limited to the (human-based) pondering of a confusion matrix-representation like the one depicted in figure 1, where the warmer, brighter (resp. cooler, darker) colour hues are designed to be related to high occurrence (resp. to low occurrence) of events. Often, this type of analysis is used

\footnotetext{
* This work has been partially supported by two grants for "Estancias de Tecnólogos Españoles en el International Computer Science Institute, año 2006" of the Spanish Ministry of Industry and a Spanish Government-Comisión Interministerial de Ciencia y Tecnología project TEC2005-04264/TCM.
} 


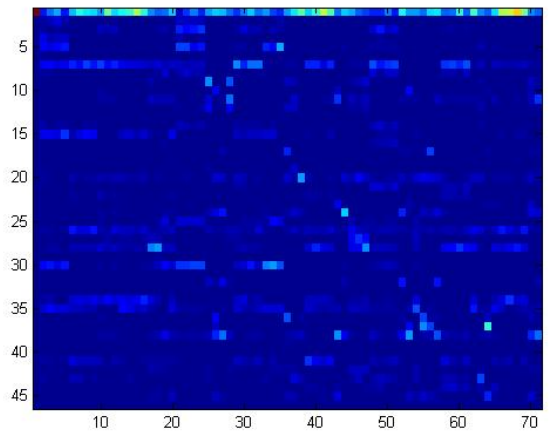

Fig. 1. Confusion matrix of the desired transformation of English phoneme labels of speech frames versus their true Mandarin phoneme labels

to bootstrap existing classifiers in order to obtain even better classification figures or simply to understand the underlying principles of the methods employed in designing the classification. In particular, in speech recognition, the designer of a system is challenged to find in this type of representation meaningful or systematic confusions to determine to what degree the behaviour of an automatic system differs from human performance.

$\mathcal{K}$-Formal Concept Analysis was introduced in [1] as a generalisation of standard Formal Concept Analysis in the sense that incidences $R \in \mathcal{K}^{n \times p}$ represented as matrices may take values in an idempotent, reflexive semifield $\mathcal{K}$ and we take $R(i, j)=\lambda$ to mean "object $g_{i}$ has attribute $m_{j}$ in degree $\lambda$." Adequate analogues of basic objects in Formal Concept Analysis become therefore available.

Two serious obstacles may prevent widespread adoption of $\mathcal{K}$-Formal Concept Analysis as a data exploration technique complementary to the standard theory: on the one hand, the $\mathcal{K}$-Formal Concept Analysis analogue of the main theorem of Formal Concept Analysis is incomplete and this may worry the user willing to be on a sound mathematical ground; on the other hand, [1] did not provide an algorithm for constructing the lattice of a $\mathcal{K}$-valued formal context, which prevents its use as a data-intensive exploration procedure.

In this paper, we try to explore further whether $\mathcal{K}$-Formal Concept Analysis is a proper generalisation of standard Formal Concept Analysis for finite contexts and to pave the way for the completion of the main theorem. In order to do so we introduce the structural lattice of a $\mathcal{K}$-Formal Context and try to relate it to the Concept Lattice of a Formal Context.

In section 2 we first review the theory of idempotent semirings and their semimodules with a view to providing the necessary objects for our discussion. In section 3.1 we present a summary of the theory of $\mathcal{K}$-Formal Concept Analysis presented in $([1], \S .3)$ and add a new theoretical construct, the structural lattice of a semimodule over an idempotent, reflexive semiring. We demonstrate in section 4 the use of this new tool to analyse confusion matrices of multiple 
input-multiple output classifiers, which turn out to be amenable to $\mathcal{K}$-Formal Concept Analysis modelling, and finish with a summary of contributions and an outlook.

\section{Mathematical Preliminaries: semimodules over idempotent, reflexive semifields as vector spaces}

\subsection{Idempotent Semirings}

A semiring $\mathcal{K}=\langle K, \oplus, \otimes, \epsilon, e\rangle$ is an algebraic structure whose additive structure, $\langle K, \oplus, \epsilon\rangle$, is a commutative monoid and the multiplicative one, $\langle K, \otimes, e\rangle$, a monoid whose multiplication distributes over addition from right and left and whose neutral element is absorbing for $\otimes, \quad \epsilon \otimes x=\epsilon, \forall x \in K[2]$. On any semiring $\mathcal{K}$ left and right multiplications can be defined:

$$
\begin{array}{lrl}
L_{a}: K \rightarrow K & R_{a}: K \rightarrow K \\
b \mapsto L_{a}(b)=a b & b \mapsto R_{a}(b)=b a
\end{array}
$$

A commutative semiring is a semiring whose multiplicative structure is commutative, and a semifield one whose multiplicative structure over $K \backslash\{\epsilon\}$ is a group. Thus, compared to a ring, a semiring which is not a ring lacks additive inverses.

An idempotent semiring $\mathcal{K}$ is a semiring whose addition is idempotent: $\forall a \in$ $K, a \oplus a=a$. All idempotent commutative monoids $(K, \oplus, \epsilon)$ are endowed with a natural order $\forall a, b \in K, a \leq b \Longleftrightarrow a \oplus b=b$, which turns them into join-semilattices with least upper bound defined as $a \vee b=a \oplus b$. Moreover, for the additive structure of and idempotent semiring $\mathcal{K}$ the neutral element is the infimum for this natural order, $\epsilon_{\mathcal{K}}=\perp$.

An idempotent semiring $\mathcal{K}$ is complete, if it is complete as a naturally ordered set and left $\left(L_{a}\right)$ and right $\left(R_{a}\right)$ multiplications are lower semicontinuous, that is, they commute with joins over any subset of $\mathcal{K}$. Therefore, complete idempotent semirings, as join-semilattices with infimum are automatically complete lattices [3] with join $(\vee, \max$ or sup) and meet $(\wedge$, min or inf) connected by the equivalences: $\forall a, b \in K, a \leq b \Longleftrightarrow a \vee b=b \Longleftrightarrow a \wedge b=a$.

Example 1. 1. The Boolean semiring $\mathcal{B}=\langle\mathbb{B}, \vee, \wedge, 0,1\rangle$, with $\mathbb{B}=\{0,1\}$, is complete, idempotent and commutative.

2. The completed Maxplus semiring $\bar{R}_{\max ,+}=\langle\mathbb{R} \cup\{ \pm \infty\}$, $\max ,+,-\infty, 0\rangle$, is a complete, idempotent semifield when defining $-\infty+\infty=-\infty$, so that $\epsilon_{\mathcal{K}} \otimes \top_{\mathcal{K}}=\epsilon_{\mathcal{K}}$ for $\mathcal{K} \equiv \bar{R}_{\text {max },+}$

3. The completed Minplus semiring $\bar{R}_{\text {min },+}=\langle\mathbb{R} \cup\{ \pm \infty\}$, min, $+, \infty, 0\rangle$ is a complete, idempotent semifield with a similar completion to that of ex. 2 with $\infty+(-\infty)=\infty$, that is $\epsilon_{\mathcal{K}} \otimes \top_{\mathcal{K}}=\epsilon_{\mathcal{K}}$ for $\mathcal{K} \equiv \bar{R}_{\text {min },+}$.

\subsection{Idempotent Semimodules: Basic Definitions}

A semimodule over a semiring is defined in a similar way to a module over a ring $[4,5,6]^{1}$ : a left $\mathcal{K}$-semimodule, $\mathcal{X}=\left\langle X, \oplus, \epsilon_{X}\right\rangle$, is an additive commutative

\footnotetext{
${ }^{1}$ We are following essentially the notation of [4].
} 
monoid endowed with a map $(\lambda, x) \mapsto \lambda \cdot x$ such that for all $\lambda, \mu \in K, x, z \in X$, and following the convention of dropping the symbol for the scalar action and multiplication for the semiring we have:

$$
\begin{aligned}
(\lambda \mu) x & =\lambda(\mu x) & & \epsilon_{K} x=\epsilon_{X} \\
\lambda(x \oplus z) & =\lambda x \oplus \lambda z & & e_{K} x=x
\end{aligned}
$$

The definition of a right $\mathcal{K}$-semimodule, $\mathcal{Y}$, follows the same pattern with the help of a right action, $(\lambda, y) \mapsto y \lambda$ and similar axioms to those of (2.) A $(\mathcal{K}, \mathcal{S})$-semimodule is a set $M$ endowed with left $\mathcal{K}$-semimodule and a right $\mathcal{S}$ semimodule structures, and a $(\mathcal{K}, \mathcal{S})$-bisemimodule a $(\mathcal{K}, \mathcal{S})$-semimodule such that the left and right multiplications commute. For a left $\mathcal{K}$-semimodule, $\mathcal{X}$, the left and right multiplications are defined as:

$$
\begin{array}{ll}
L_{\lambda}^{\mathcal{K}}: X \rightarrow X & R_{x}^{\mathcal{X}}: K \rightarrow X \\
x \mapsto L_{\lambda}^{\mathcal{K}}(x)=\lambda x & \lambda \mapsto R_{x}^{\mathcal{X}}(\lambda)=\lambda x
\end{array}
$$

And similarly, for a right $K$-semimodule. If $\mathcal{X}, \mathcal{Z}$ are left semimodules a morphism of left semimodules or left linear map $F: \mathcal{X} \rightarrow \mathcal{Z}$ is a map that preserves finite sums and commutes with the action: $F(\lambda v \oplus \mu w)=\lambda F(v) \oplus \mu F(w)$, and similarly, mutatis mutandis for right linear maps of right semimodules.

The elements of a semimodule may be conceived as vectors ${ }^{2}$. Given a semiring $\mathcal{K}$ and a left $\mathcal{K}$-semimodule $\mathcal{X}$, for each finite, non-void set $W \subseteq X$, there exists an homomorphism $\alpha: K^{W} \rightarrow X, f \mapsto \bigoplus_{w \in W} f(w) w$. Moreover, $\alpha$ induces a congruence of semimodules $\equiv_{\alpha}$ on $K^{W}$, by $f \equiv_{\alpha} g \Longleftrightarrow \alpha(f)=\alpha(g)$. Then $W$ is a set of generators or a generating family precisely when $\alpha$ is surjective, in which case any element $x \in X$ can be written as $x=\bigoplus_{w \in W} \lambda_{w} w$, and we will write $\mathcal{X}=\langle W\rangle_{\mathcal{K}}$, that is, $\mathcal{X}$ is the span of $W$. A semimodule is finitely generated if it has a finite set of generators.

For individual vectors, we say that $x \in W$ is dependent (in $W$ ) if $x=$ $\bigoplus_{w \in W \backslash\{x\}} \lambda_{w} w$ otherwise, we say that it is free (in $W$ ). The set $W$ is linearly independent if and only if $\equiv_{\alpha}$ is the trivial congruence, that is, when $\bigoplus_{w \in W} f(w) w=\bigoplus_{w \in W} h(w) w \Longleftrightarrow f=h$, otherwise, $W$ is linearly dependent. Let $\operatorname{ker} \alpha=\left\{f \in K^{W} \mid \alpha(f)=0\right\}$; then $W$ is weakly linearly independent if and only if ker $\alpha=\{0\}$, otherwise it is weakly linearly dependent.

A basis for $\mathcal{X}$ (over $\mathcal{K}$ ) is a linearly-independent set of generators, and a semimodule generated by a basis is free. By definition, in a free semimodule $\mathcal{X}$ with with basis $\left\{x_{i}\right\}_{i \in I}$ each element $x \in X$ can be uniquely written as $x=\bigoplus_{i \in I} \alpha_{i} x_{i}$, with $\left[a_{i}\right]_{i \in I}$ the co-ordinates of $x$ with respect to the basis. A weakly linearly-independent set of generators for $\mathcal{X}$ is a weak basis for $\mathcal{X}$ (over $\mathcal{K})$. The cardinality of a (weak) basis is the (weak) rank of the semimodule.

In this framework, notions in usual vector spaces have to be imported with care. For instance, the image of a linear map $F: \mathcal{X} \rightarrow \mathcal{Y}$ is simply the semimodule $\operatorname{Im} F=\{F(x) \mid x \in X\}$, but it is in general not free.

\footnotetext{
${ }^{2}$ Most of the material in this section is from [5], $\S 17$, and $[7,8,9]$.
} 
Given a free semimodule $\mathcal{X}$ with basis $\left\{x_{i}\right\}_{i \in I}$, for each family $\left\{y_{i}\right\}_{i \in I}$ of elements of an arbitrary semimodule $\mathcal{Y}$ there is a unique morphism of semimodules $F: \mathcal{X} \rightarrow \mathcal{Y}$ such that $F\left(x_{i}\right)=y_{i}, \forall i \in I$, namely $F\left(\bigoplus_{i \in I} \lambda_{i} x_{i}\right)=\bigoplus_{i \in I} \lambda_{i} y_{i}$ and all the linear maps $\operatorname{Lin}(\mathcal{X}, \mathcal{Y})$ are obtained in this way ([7], prop. $\S 73$; [5], prop. $\$ 17.12)$. That is, linear maps from free semimodules are characterised by the images of the elements of a basis.

On the other hand, a semiring $\mathcal{K}$ has the linear extension property if for all free, finitely generated $\mathcal{K}$-semimodules $\mathcal{X}, \mathcal{Y}$, for all finitely generated subsemimodules $\mathcal{Z} \subset X$ and for all $F \in \operatorname{Lin}(\mathcal{Z}, \mathcal{Y})$, there exists $H \in \operatorname{Lin}(\mathcal{X}, \mathcal{Y})$ such that $\forall x \in X, H(x)=F(x)$. The importance of this property derives from the fact that when the linear extension property holds, each linear map between finitely generated subsemimodules of free semimodules is represented by a matrix. In particular, when it holds for free, finitely generated (left) semimodules, $\mathcal{X}$ and $\mathcal{Y}$ with bases $\left\{x_{i}\right\}_{i \in I}$ and $\left\{y_{j}\right\}_{j \in J}$, each linear map is characterised by the $n \times p$-matrix $R=\left(F\left(x_{i}\right)_{j}\right)$, which sends vector $x=\left\{x_{i}\right\}_{i=1}^{n}$ to the vector $F(x) \simeq\left((x R)_{1}, \ldots,(x R)_{p}\right)$.

\subsection{Semimodules over Idempotent Semirings}

In this section all semimodules will be defined over an idempotent semifield. Recall that examples of these are $\mathcal{B}$, the Boolean semifield and the completed maxplus and minplus semifields.

Idempotency and Natural Order in Semimodules. A left, right $\mathcal{K}$-semimodule $\mathcal{X}$ over an idempotent semiring $\mathcal{K}$ inherits the idempotent law, $v \oplus v=$ $v, \forall v \in X$, which induces a natural order on the semimodule by $v \leq w \Longleftrightarrow$ $v \oplus w=w, \forall v, w \in X$ whereby it becomes a $\vee$-semilattice, with $\epsilon_{\mathcal{X}}$ the minimum. In the following we systematically equate idempotent $\mathcal{K}$-semimodules and semimodules over an idempotent semiring $\mathcal{K}$. When $\mathcal{K}$ is a complete idempotent semiring, a left $\mathcal{K}$-semimodule, $\mathcal{X}$ is complete (in its natural order) if it is complete as a naturally ordered set and its left and right multiplications are (lower semi)continuous. Trivially, it is also a complete lattice, with join and meet operations given by: $v \leq w \Longleftrightarrow v \vee w=w \Longleftrightarrow v \wedge w=v$. This extends naturally to right- and bisemimodules.

Example 2. 1. Each semiring, $\mathcal{K}$, is a left (right) semimodule over itself, with the semiring product as left (right) action. Therefore, it is a $(\mathcal{K}, \mathcal{K})$ bisemimodule over itself, because both actions commute by associativity. Such is the case for the Boolean $(\mathcal{B}, \mathcal{B})$-bisemimodule, the Maxplus and the Minplus bisemimodules. These are all complete and idempotent.

2. For $n, m \in \mathbb{N}$, the set of matrices $K^{n \times p}$ is a $\left(K^{n \times n}, K^{p \times p}\right)$-bisemimodule with matrix multiplication-like left and right actions and component-wise addition, the set of column vectors $K^{p \times 1}$ is a $\left(K^{p \times p}, K\right)$-bisemimodule and the set of row vectors $K^{1 \times n}$ a $\left(K, K^{n \times n}\right)$-bisemimodule with similarly defined operations. If $\mathcal{K}$ is idempotent (resp. complete), then all are idempotent (resp. complete) with the component-wise partial order their natural order. 
As in the semiring case, because of the natural order structure, the actions of idempotent semimodules admit residuation: given a complete, idempotent left $\mathcal{K}$-semimodule, $\mathcal{X}$, we define for all $x, z \in X, \lambda \in K$ the residuals:

$$
\begin{array}{ll}
\left(L_{\lambda}^{\mathcal{K}}\right)^{\#}: X \rightarrow X & \left(L_{\lambda}^{\mathcal{K}}\right)^{\#}(z)=\bigvee\{x \in X \mid \lambda x \leq z\}=\lambda \backslash z \\
\left(R_{x}^{\mathcal{X}}\right)^{\#}: X \rightarrow K & \left(R_{x}^{\mathcal{X}}\right)^{\#}(z)=\bigvee\{\lambda \in K \mid \lambda x \leq z\}=z / x
\end{array}
$$

and likewise for a right semimodule, $\mathcal{Y}$.

There is a remarkable operation that changes the character of a semimodule while at the same time reversing its order by means of residuation:

Definition 3. Let $\mathcal{K}$ be a complete, idempotent semiring, and $\mathcal{Y}$ be a complete right $\mathcal{K}$-semimodule, its opposite semimodule is the complete left $\mathcal{K}$-semimodule $\mathcal{Y}^{o p}=\langle Y, \stackrel{o p}{\oplus, \stackrel{o p}{\rightarrow}}\rangle$ with the same underlying set $Y$, addition defined by $(x, y) \mapsto$ $x \stackrel{o p}{\oplus} y=x \wedge y$ where the infimum is for the natural order of $\mathcal{Y}$, and left action:

$$
K \times Y \rightarrow Y \quad(\lambda, y) \mapsto \lambda \stackrel{o p}{\rightarrow} y=y / \lambda
$$

Consequently, the order of the opposite is the dual of the original order.

For the opposite semimodule the residual definitions are:

$$
\begin{aligned}
& \lambda \backslash x=\left(L_{\lambda}^{\mathcal{Y}^{o p}}\right)^{\#}(x)=\bigwedge\{y \in Y \mid x \leq y / \lambda\}=x \cdot \lambda \\
& x / y=\left(R_{y}^{\mathcal{Y}^{o p}}\right)^{\#}(x)=\bigvee\{\lambda \in K \mid x \leq y / \lambda\}=x \backslash y
\end{aligned}
$$

Note that we can define mutatis mutandis the opposite semimodule of a left $\mathcal{K}$-semimodule, $\mathcal{X}$, with right action $x \stackrel{o p}{\longleftarrow} \lambda=\lambda \backslash x$. Also, noticing that the first residual in eq. 5 is in fact an involution we may conclude that the operation of finding the opposite of a complete (left, right) $\mathcal{K}$-semimodule is an involution: $\left(\mathcal{Y}^{o p}\right)^{o p}=\mathcal{Y}$.

Constructing Galois Connections in Idempotent Semimodules. The following construction is due to Cohen et al. [4]. Let $\mathcal{K}$ be a complete idempotent semiring; for a bracket $\langle\cdot \mid \cdot\rangle: X \times Y \rightarrow Z$ between left and right $\mathcal{K}$-semimodules, $\mathcal{X}$ and $\mathcal{Y}$ respectively, onto a $\mathcal{K}$-bisemimodule $\mathcal{Z}$ and an arbitrary element $\varphi \in Z$, which we call the pivot, define the maps:

$$
\begin{array}{ll}
\bar{\varphi}_{\varphi}^{-}: X \rightarrow Y & x_{\varphi}^{-}=L_{x}^{\#}(\varphi)=\bigvee\{y \in Y \mid\langle x \mid y\rangle \leq \varphi\} \\
\bar{\varphi} \cdot: Y \rightarrow X & \bar{\varphi}_{\varphi} y=R_{y}^{\#}(\varphi)=\bigvee\{x \in X \mid\langle x \mid y\rangle \leq \varphi\}
\end{array}
$$

We have $\langle x \mid y\rangle \leq \varphi \Longleftrightarrow y \leq x_{\varphi}^{-} \Longleftrightarrow x \leq{ }_{\varphi} y$, whence the pair is a Galois connection between $\mathcal{Y}$ and $\mathcal{X},(\cdot \bar{\varphi}, \bar{\varphi} \cdot): \mathcal{X} \multimap \mathcal{Y}$. This construction is affected crucially by the choice of a suitable pivot $\varphi$ : if we consider the bracket 
to reflect a degree of relatedness between the elements of each pair, only those pairs $(x, y) \in X \times Y$ are considered by the connection whose degree amounts at most to $\varphi$. Therefore we can think of the pivot as a maximum degree of existence allowed for the pairs.

Recall $\mathcal{X}$ and $\mathcal{Y}$ are both complete lattices as well as free vector spaces. Note that the closure lattices $\bar{X}={ }_{\varphi}(\mathcal{Y})$ and $\underline{Y}=(\mathcal{X})_{\varphi}^{-}$do not agree with their ambient vector spaces in their joins, but only in their meets. To improve on this, the notion of a left (resp. right) reflexive, $(\mathcal{K}, \varphi)$, semiring is introduced in [4] as a complete idempotent semiring such that $(\langle\cdot \mid \cdot\rangle: K \times K \rightarrow K, \varphi)$ with $\langle\lambda \mid \mu\rangle=\lambda \mu$ induces a perfect Galois connection ${ }^{3}$ under construction (6) for all $\lambda \in K,{ }^{-}\left(\lambda^{-}\right)=\lambda$ (resp. $\left.\left({ }^{-} \lambda\right)^{-}=\lambda.\right)^{4}$ The interest in reflexive semirings stems from the fact that in such semirings $\bar{X}$ and $\bar{Y}$ are actually subsemimodules (that is their suprema coincide with those) of the corresponding spaces ([4], prop. 28).

Note that $\varphi$ need not be unique: if $(\mathcal{K}, \varphi)$ is right (or left) reflexive, for any $\lambda \in K$ invertible, $(\mathcal{K}, \varphi \lambda)$ is left reflexive (and $(\mathcal{K}, \lambda \varphi)$ is right reflexive.) Finally, Cohen et al. [4] prove that idempotent semifields are left and right reflexive, and suggest that for the Boolean semiring we must choose $\varphi=0_{\mathcal{B}}$, the bottom in the order. For other semifields any invertible element may be chosen, e.g. $\varphi=e_{\mathcal{K}}$.

Idempotent Semimodules as Vector Spaces. When $\mathcal{K}$ is an idempotent semiring if a $\mathcal{K}$-semimodule has a (weak) basis, it is unique up to a permutation and re-scaling of the axes, that is a scaling endomorphism ([9], Th. §3.1), $x_{i}^{\prime}=\lambda_{i} x_{i}$, and every finitely generated $\mathcal{K}$-semimodule has a weak basis ([9], Coroll. §3.6). In particular, let $\mathcal{K}$ be an idempotent semifield, then the free idempotent semimodule with $n$ generators is isomorphic to $\mathcal{K}^{n}$. Essentially, such free idempotent semimodules are generated by the bases $E_{n} \triangleq\left\{e_{i}\right\}_{i=1}^{n}, e_{i}=$ $\left(\delta_{i 1}, \delta_{i 2}, \ldots, \delta_{i n}\right)$, where $\delta_{i j}$ is the Kronecker symbol over $\mathcal{K}, \delta_{i i}=e_{\mathcal{K}}, \delta_{i j}=$ $\epsilon_{\mathcal{K}}, i \neq j$.

Importantly, the linear property holds in every idempotent semiring which is a distributive lattice for the natural order ([7], Th. §83). This is the case for the semifields $\mathbb{B}$ (the Boolean semiring), $\bar{R}_{\max ,+}$ and $\bar{R}_{\min ,+}$. Therefore, in such semimodules, modulo a choice of bases for $\mathcal{X}$ and $\mathcal{Y}$, we may identify $\mathcal{X} \cong \mathcal{K}^{1 \times n}$ and $\mathcal{Y} \cong \mathcal{K}^{1 \times p}$, and linear maps to matrix transformations $\operatorname{Lin}(\mathcal{X}, \mathcal{Y}) \cong \mathcal{K}^{n \times p}$, $R: \mathcal{K}^{1 \times n} \rightarrow \mathcal{K}^{1 \times p}, x \mapsto x R$. When passing from left to right semimodules this should read $\mathcal{K}^{p \times 1} \rightarrow \mathcal{K}^{n \times 1}, y \mapsto R y$.

Idempotent semimodules have additional properties which make them easier to work with as spaces: when $\mathcal{X}$ is a vector space over an idempotent semiring $\mathcal{K}$, for a set of vectors, $W \subseteq X$, the set of finite sums $W^{+} \triangleq\left\{\bigoplus_{i} w_{i} \mid w_{i} \in W\right\}$, is a $\vee$-subsemilattice of $\langle W\rangle_{K}$. Therefore, the $\vee$-irreducibles of $W$, generate the span of $W,\langle\mathcal{J}(W)\rangle_{\mathcal{K}}=\langle W\rangle_{\mathcal{K}}$. This makes the $\vee$-irreducibles an interesting set to obtain a basis.

\footnotetext{
3 That is, a pair of mutually inverse isomorphisms.

${ }^{4}$ When the pivot is the multiplicative unit $\varphi=e$ we drop it.
} 
The Projective Space and the Structural Semilattice. Let $\mathcal{X}$ be a left $\mathcal{K}$-semimodule over an idempotent semiring $\mathcal{K}$. The relation $x \preccurlyeq y \Leftrightarrow \exists \lambda \in$ $K, x \leq \lambda \otimes y$ defines a quasi-order $\langle\mathcal{X}, \preccurlyeq\rangle$. Since any basis $W_{\mathcal{X}}$ is unique up to a re-scaling map, the Hasse diagram of $\left(W_{\mathcal{X}}, \preccurlyeq\right)$ is independent of the choice of basis.

Now define the equivalence relation ([7], p. 41), $x \simeq y \Leftrightarrow x \preccurlyeq y$ and $y \preccurlyeq x$. This relation appears already in ([10], p. 2018) and was later considered under the name of siblinghood relation [11] where two vectors $v$ and $w$ are siblings if $w=\lambda \otimes v$ for some $\lambda \in K$. This is a congruence of $\vee$-semilattices, therefore [7], the projective space is the quotient set $\mathbb{P}(\mathcal{X}) \triangleq\left\{[x]_{\simeq} \mid x \in X\right\}$ (where $[x]_{\simeq}$ the equivalence class of $x \in X$, is also called the ray of $x$ or the sibling class of $x$ ), which is also a $\vee$-semilattice, $\langle\mathbb{P}(\mathcal{X}), \preccurlyeq\rangle$ with the induced order.

For any subset $W \subseteq X$, let a section of the quotient set $W / \simeq, \sigma: 2^{X} \rightarrow$ $X, W \mapsto \sigma(W)$ be a set obtained by choosing a single representative from each sibling class. Note that a section has the order directly induced by $\preccurlyeq_{\mathcal{X}}$ [11] . It is now clear that a section of the quotient set of the join irreducibles of a set of vectors is a (weak) basis of their span $\sigma[\mathcal{J}(W)]=\langle W\rangle_{\mathcal{K}}$.

Next, consider the siblinghood relation above and a basis $W_{\mathcal{X}}$ :

Definition 4 (Wagneur [10]). Let $\mathcal{X}$ be a left $\mathcal{K}$-semimodule over and idempotent semifield $\mathcal{K}$ with a basis $W_{\mathcal{X}}$. The structural $(\vee$-)semilattice of $\mathcal{X}, S(\mathcal{X})$ is the quotient set of $W_{\mathcal{X}}{ }^{+}$through the siblinghood relation $S(\mathcal{X}) \triangleq W_{\mathcal{X}}{ }^{+} / \simeq$.

The following theorem states that the quotient set $W_{\mathcal{X}}{ }^{+} / \simeq$ is an intrinsic invariant of $\mathcal{X}$.

Theorem 1 ([10], Th. 2). For any basis $W_{\mathcal{X}}$ of a left $\mathcal{K}$-semimodule over and idempotent semifield $\mathcal{K}$, the quotient map $\pi: W_{\mathcal{X}}{ }^{+} \rightarrow W_{\mathcal{X}}{ }^{+} / \simeq, w \mapsto[w]_{\simeq}$ is an epimorphism of $\vee$-semilattices and $W_{\mathcal{X}}{ }^{+} / \simeq$ is independent of the particular choice of basis $W_{\mathcal{X}}$.

Since $\pi$ is an epimorphism of $\vee$-semilattices and $\simeq$ a $\vee$-congruence, the quotient set of the basis through the siblinghood relation $W_{\mathcal{X}} / \simeq=\left\{[w]_{\simeq} \mid w \in W_{\mathcal{X}}\right\}$ is the set of $\vee$-irreducibles of the quotient set, $\mathcal{J}\left(W_{\mathcal{X}}{ }^{+} / \simeq\right)=W_{\mathcal{X}} / \simeq[10]$.

\section{The Structural Lattice of a $\mathcal{K}$-Concept Lattice}

\section{$3.1 \quad \mathcal{K}$-Formal Concept Analysis, a Reminder}

The following has been adapted from [1] to emphasise the fact that the theory does not cover the case of unbounded cardinalities ${ }^{5}$.

Definition 5 ( $\mathcal{K}$-valued formal context ). For $n, p \in \mathbb{N}$, given two sets of objects $G=\left\{g_{i}\right\}_{i=1}^{n}$, and attributes $M=\left\{m_{j}\right\}_{j=1}^{p}$, an idempotent semiring, $\mathcal{K}$, and a $\mathcal{K}$-valued matrix, $R \in \mathcal{K}^{n \times p}$, where $R(i, j)=\lambda$ reads as "object $g_{i}$ has attribute $m_{j}$ in degree $\lambda$ " and dually "attribute $m_{j}$ is manifested in object $g_{i}$ to degree $\lambda$ ", the triple $(G, M, R)_{\mathcal{K}}$ is called a $\mathcal{K}$-valued formal context.

\footnotetext{
${ }^{5}$ This section follows in the tracks of $\S 1.1$ of [12].
} 
Clearly single objects are isomorphic to elements of the space $\mathcal{K}^{1 \times p}$, that is rows of $R$ or object descriptions, vectors of as many values as attributes. And dually, single attributes are isomorphic to elements of the space $\mathcal{K}^{n \times 1}$, columns of $R$ or attribute descriptions. We model $(\mathcal{K}$-valued) sets of objects as row vectors in a left $\mathcal{K}$-semimodule, $x \in \mathcal{X} \cong \mathcal{K}^{1 \times n}$, and sets of attributes as column vectors in a right $\mathcal{K}$-semimodule, $y \in \mathcal{Y} \cong \mathcal{K}^{p \times 1}$ as generalisations of characteristic functions in the power sets $\mathbf{2}^{G}, \mathbf{2}^{M}$, respectively.

The proof of the following proposition is crucial for future argumentation, hence we reproduce it in full:

Proposition 2. Let $(\mathcal{K}, \varphi)$ be a reflexive, idempotent semiring. For a $\mathcal{K}$-valued formal context $(G, M, R)_{\mathcal{K}}$, with $n, p \in \mathbb{N}$, there is at least one Galois connection between the lattices of $\left(\mathcal{K}\right.$-valued) sets of objects $\mathcal{K}^{1 \times n}$ and attributes $\mathcal{K}^{p \times 1}$.

Proof. Recall that $\mathcal{X}=\mathcal{K}^{1 \times n}$ is a left semimodule and $\mathcal{Y}=\mathcal{K}^{p \times 1}$ a right semimodule, whence $\mathcal{X}^{o p}$ and $\mathcal{Y}^{o p}$ are right and left semimodules, respectively, whose multiplications are $R \stackrel{o p}{\longleftarrow} x=x^{t} \backslash R$ and $y \stackrel{o p}{\rightarrow} R=R / y^{t}$. We build a new bracket over the opposite semiring $\mathcal{K}^{o p}$ as given by $\langle y \mid x\rangle_{R}=y \stackrel{o p}{\rightarrow} R \stackrel{o p}{\longleftarrow} x=x^{t} \backslash R / y^{t}$. Therefore, by the construction of section 2.3 the following maps form a Galois connection $(\cdot \bar{\varphi}, \bar{\varphi} \cdot): \mathcal{Y}^{o p} \multimap \mathcal{X}^{o p}:$

$$
\begin{aligned}
& y_{\varphi}^{-}=\bigwedge\left\{x \in X \mid\langle y \mid x\rangle_{R} \geq \varphi\right\}=(y \stackrel{o p}{\rightarrow} R) \bigwedge^{o p} \varphi \\
& \bar{\varphi}^{-} x=\bigwedge\left\{y \in Y \mid\langle y \mid x\rangle_{R} \geq \varphi\right\}=\varphi /(R \stackrel{o p}{\longleftarrow} x)
\end{aligned}
$$

In fact, in an idempotent semifield we are guaranteed enough $\varphi$ to build as many connections as necessary: choose any invertible $\lambda \in K$, so that $\varphi=\lambda \otimes e_{\mathcal{K}}$.

Definition 6 ( $\varphi$-polars). Given a reflexive, idempotent semiring $(\mathcal{K}, \varphi)$ and a $\mathcal{K}$-valued formal context $(G, M, R)_{\mathcal{K}}$ satisfying the conditions of proposition 2 , we call $\varphi$-polars the dually adjoint maps of the corresponding Galois connection of equation (\%)

However, in this dualised construction the pivot describes a minimum degree of existence required for pairs $(x, y) \in X \times Y$ to be considered for operation.

Definition 7 (Formal $\varphi$-concepts and $\varphi$-Concept Lattices). Given a reflexive, idempotent semiring $(\mathcal{K}, \varphi)$, a $\mathcal{K}$-valued formal context $(G, M, R)_{\mathcal{K}}$ with $n, p \in \mathbb{N}$, and $\mathcal{K}$-valued vector spaces of rows $\mathcal{X} \cong \mathcal{K}^{1 \times n}$ and columns $\mathcal{Y} \cong \mathcal{K}^{p \times 1}$

1. A (formal) $\varphi$-concept of the formal context $(G, M, R)_{\mathcal{K}}$ is a pair $(a, b) \in$ $\mathcal{X} \times \mathcal{Y}$ such that ${ }_{\varphi}^{-} a=b$ and $b_{\varphi}^{-}=a$. We call $a$ the extent and $b$ the intent of the concept $(a, b)$, and $\varphi$ its (minimum) degree of existence.

2. If $\left(a_{1}, b_{1}\right)\left(a_{2}, b_{2}\right)$ are $\varphi$-concepts of a context, they are ordered by the relation $\left(a_{1}, b_{1}\right) \leq\left(a_{2}, b_{2}\right) \Longleftrightarrow a_{1} \leq a_{2} \Longleftrightarrow b_{1} \stackrel{o p}{\leq} b_{2}$, called the hierarchical order. The set of all concepts ordered in this way is called the $\varphi$-concept lattice, $\underline{\mathfrak{B}}^{\varphi}(G, M, R)_{\mathcal{K}}$, of the $\mathcal{K}$-valued context $(G, M, R)_{\mathcal{K}}$ 
The nomenclature introduced in definition 7 is supported by the following:

Theorem 3 (Fundamental theorem of $\mathcal{K}$-valued Formal Concept Analysis, finite version, $1^{\text {st }}$ half). Given a reflexive, idempotent semiring $(\mathcal{K}, \varphi)$, the $\varphi$-concept lattice $\underline{\mathfrak{B}}^{\varphi}(G, M, R)_{\mathcal{K}}$ of a $\mathcal{K}$-valued formal context $(G, M, R)_{\mathcal{K}}$ with $n, p \in \mathbb{N}$, is a (finite, complete) lattice in which infimum and supremum are given by:

$$
\bigwedge_{t \in T}\left(a_{t}, b_{t}\right)=\left(\bigoplus_{t \in T}^{o p} a_{t},{ }_{\varphi}^{-}\left[\bigoplus_{t \in T}^{o p} a_{t}\right]\right) \bigvee_{t \in T}\left(a_{t}, b_{t}\right)=\left(\left[\bigoplus_{\mathrm{t} \in \mathrm{T}}^{\mathrm{op}} b_{t}\right]_{\varphi}^{-}, \bigoplus_{t \in T}^{o p} b_{t}\right)
$$

In [1] the question was posed whether this theorem could be completed in the direction of $\S 1.1$ of [12]. This will be looked into next.

\subsection{The Structural Lattice of a $\mathcal{K}$-Concept Lattice}

This section contains this paper's theoretical contributions to the characterisation of the semimodules over an idempotent, reflexive semifield $\mathcal{K}$ that allow to define the anti-isomorphic lattices of theorem 3.

From definition 4 and theorem 1 the notion of structural semilattice emerges as important to characterise semimodules over an idempotent semifield. We may wonder whether more interesting characterisations may be possible when the set of generators comes from a homomorphism of spaces of finite dimension.

For that purpose, recall that in the Galois connection of equation $(7),\left({ }^{-}, \bar{\varphi} \cdot\right)$ : $\mathcal{Y}^{o p} \multimap \mathcal{X}^{o p}$, the dually isomorphic closure lattices are:

$$
\overline{\mathcal{Y}^{o p}}=\{\varphi \stackrel{o p}{\mid}(R \stackrel{o p}{\leftarrow} x) \mid x \in X\} \quad \overline{\mathcal{X}^{o p}}=\{(y \stackrel{o p}{\rightarrow} R) \stackrel{o p}{\backslash} \varphi \mid y \in Y\}
$$

where $\mathcal{X}$ is the free space of object sets and $\mathcal{Y}$ is the free space of attribute sets. Now let the singleton sets of objects (row vectors), $\boldsymbol{g}_{i}=\left[\epsilon \cdots e_{i} \cdots \epsilon\right]$, and attributes (column vectors), $\boldsymbol{m}_{j}=\left[\epsilon \cdots e_{j} \cdots \epsilon\right]^{T}$, which are bases of their respective spaces, be mapped through the polars $W_{\overline{\mathcal{Y}^{o p}}} \triangleq{ }_{\varphi}^{-}\left(\left\{g_{i}\right\}_{i=1}^{n}\right)$ and $W_{\overline{\mathcal{X}^{o p}}} \triangleq$ $\left(\left\{m_{j}\right\}_{j=1}^{p}\right)_{\varphi}^{-}$, to obtain generator sets for the closure lattices: $\left\langle W_{\overline{\mathcal{Y}^{o p}}}\right\rangle_{\mathcal{K}^{o p}}=\overline{\mathcal{Y}^{o p}}$ $\left\langle W_{\overline{\mathcal{X}^{o p}}}\right\rangle_{\mathcal{K}^{o p}}=\overline{\mathcal{X}^{o p}}$.

But note that the generation process is directed by the algebra of the opposite semiring $\mathcal{K}^{o p}$, that is, the generation process is carried out using the addition in the opposite semimodules, $\stackrel{\circ p}{\oplus}=\wedge$. As we know that the $\vee$-irreducibles are included in each set of generators we may test the latter to find the former:

$$
\mathcal{J}\left(\overline{\mathcal{Y}^{o p}}\right) \subseteq\left\langle W_{\overline{\mathcal{Y}^{o p}}}\right\rangle_{\mathcal{K}^{o p}}=\overline{\mathcal{Y}^{o p}} \quad \mathcal{J}\left(\overline{\mathcal{X}^{o p}}\right) \subseteq\left\langle W_{\overline{\mathcal{X}^{o p}}}\right\rangle_{\mathcal{K}^{o p}}
$$

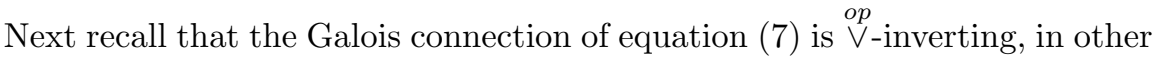
words, $\wedge$-inverting, therefore, the images of the $\stackrel{o p}{\vee}$-irreducibles are $\stackrel{o p}{\wedge}$-irreducibles:

$$
\mathcal{M}\left(\overline{\mathcal{X}^{o p}}\right)=\left(\mathcal{J}\left(\overline{\mathcal{Y}^{o p}}\right)\right)_{\varphi}^{-} \quad \mathcal{M}\left(\overline{\mathcal{Y}^{o p}}\right)={ }_{\varphi}^{-}\left(\mathcal{J}\left(\overline{\mathcal{X}^{o p}}\right)\right)
$$


Alternatively we may think of the product of each pair of join- and meetirreducible sets as being comprised of $\vee$-irreducible concepts $\wedge$-irreducible concepts with definitions resembling those of the standard theory:

$$
\tilde{\gamma}^{\varphi}\left(\boldsymbol{g}_{i}\right)=\left(\left(\bar{\varphi}\left(\boldsymbol{g}_{i}\right)\right)_{\varphi}^{-}, \bar{\varphi}\left(\boldsymbol{g}_{i}\right)\right) \quad \tilde{\mu}^{\varphi}\left(\boldsymbol{m}_{j}\right)=\left(\left(\boldsymbol{m}_{j}\right)_{\varphi}^{-}, \bar{\varphi}\left(\left(\boldsymbol{m}_{j}\right)_{\varphi}^{-}\right)\right)
$$

Definition 8 (Structural Lattice). The structural lattice $\underline{\mathfrak{B}}\left(G, M, I_{R}^{\varphi}\right)$ of a $\mathcal{K}$-Concept Lattice $\underline{\mathfrak{B}}^{\varphi}(G, M, R)$ is the Concept Lattice of the context, $\left(G, M, I_{R}^{\varphi}\right)$, where

$$
I_{R}^{\varphi}(i, j)=\tilde{\gamma}^{\varphi}\left(\boldsymbol{g}_{i}\right) \leq \tilde{\mu}^{\varphi}\left(\boldsymbol{m}_{j}\right)
$$

Note that we have not used the siblinghood relation to define the structural lattice. The coalescing of different join- or meet-irreducibles in the same ray to obtain a basis is in this case counterproductive because in any section of a partitioned $\mathcal{M}\left(\overline{\mathcal{X}^{o p}}\right), \mathcal{J}\left(\overline{\mathcal{X}^{o p}}\right)$ or their images some join- or meet-irreducibles may be missing, for instance if structural lattice has the appearance of the $N_{5}$ lattice. We believe this is one more instance of the differences between idempotent semimodules and traditional vector spaces.

\section{Example : The Analysis of Cross-Lingual Classifier Adaptation Systems}

In this example we analyse a particular problem: the cross-lingual adaptation of an automatic speech recogniser trained to recognise English phonemes into a system capable of recognising Mandarin phonemes. Our aim in this task is to analyse several ways of mapping the English outputs of such classifiers into Mandarin phonemes by observing whether the mapping has an intuitive, meaningful structure. We will compare two ways to accomplish this:

- An original system trained with English-speech data with a particular classifier-building technique.

- An enhanced system which uses the previous system as a start point and improved afterwards by using some Mandarin-speech data to learn to map the English outputs into Mandarin phonemes.

In both cases, the input to our algorithm will be the confusion matrix (more properly called the translation matrix, $T$, in this context) between English phonemes (outputs, $n=46$ ) and Mandarin phonemes (inputs, $p=71$ ) by observing the English labels that both networks assign to the Mandarin speech frames and confronting it with the true Mandarin labels.

\subsection{Lattice Construction}

This section describes the algorithm employed to obtain the structural lattice of relation $T$ for a range of degrees of existence $\varphi$ as defined in previous sections. 
For that purpose, we first transform the event counts of the confusion matrix into a maximum-likelihood estimate of the probability of the true Mandarin label $g_{i}$ given that the output of the classifier is the English label $m_{j}, P\left(G=g_{i}\right.$ | $\left.M=m_{j}\right)$. We then take logarithms to transform probabilities in $[0,1]$ into loglikelihoods, that is $\overline{\mathbb{R}}_{\text {max },+}$ costs to obtain $R \in \overline{\mathbb{R}}_{\max ,+}^{n \times p}$ The following algorithm then obtains the structural lattice of the cost matrix, $R$ :

Step 1 Compute the closures of the $n$ unitary row vectors of dimension $1 \times n$, $\boldsymbol{g}_{i}=\left[\epsilon \cdots e_{i} \cdots \epsilon\right]$ and $p$ unitary column vectors of dimension $p \times 1$, $\boldsymbol{m}_{j}=\left[\epsilon \cdots e_{j} \cdots \epsilon\right]^{T}$ that stand for the characteristic functions of singleton sets of objects and attributes, respectively. The $\varphi$-polars of definition 6 allow us to obtain, the $\vee$ - and $\wedge$-irreducible concepts of the structural lattice using equation (12).

Step 2 Build the standard context associated to those concepts and the structural lattice by comparing the previous concepts, $\underline{\mathfrak{B}}\left(G, M, I_{R}^{\varphi}\right)$ where $I_{R}^{\varphi}$ is the incidence with $\{0,1\}$ entries in formula (13.)

Step 3 Once the standard context adequate for the structural lattice is obtained for each particular $\varphi$ we used CONEXP [13] to obtain the standard lattices.

Because the Galois connection that obtains the formal concepts depends on the pivot, $\varphi$, typically the above algorithm must be carried out a number of times, one for each choice of $\varphi$ that is deemed interesting.

\subsection{Lattice Exploration}

The Influence of the Enhancement stage: Choosing $\varphi$. Our aim now is to explore the behaviour of the $\mathcal{K}$-Concept Lattice for a particular $\mathcal{K}$-formal context with varying $\varphi$. For this purpose, we have found the standard context of the structural lattice with the algorithm above for each $\varphi$ and worked out the number of concepts resulting for the standard Formal Contexts of the original and enhanced systems. Figure 2 shows this evolution where we have chosen to sample the curve more frequently as we approach the right end (i.e. $\varphi=0$ ) by using the tangent function of a uniform sampling. A logarithmic scale has been used in the vertical axis to improve the comparison of the two curves given the notorious differences in the number of concepts of the two examples we are evaluating here.

For a perfect translation between two phonemic systems of identical cardinality, the best system would show a diagonal matrix in the $\mathcal{K}$-Formal Context, equivalent to a diamond lattice of as many $\vee$ - and $\wedge$-irreducibles as phonemes. We expect to find systems that do a worse translation further and further from this structure and with increasing concept counts. Indeed, the most significant observation we can gather from the plot above is the reduction in the number of concepts achieved by the enhanced system. We can infer, therefore, that the enhancing stage improves the translation in such direction.

We notice that the overall shapes of the curves are very similar. For smaller $\varphi$ the number of concepts remains constant for each matrix being evaluated. 


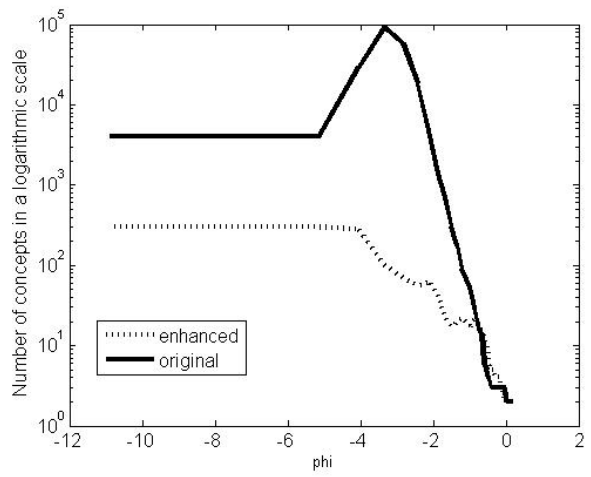

Fig. 2. Number of concepts vs. $\varphi$

For $\varphi \geq \max _{i, j} R_{i j}$, the incidence matrix is everywhere null $I_{R}=0$ leading to a two-concept lattice for both curves. In between these ranges we see how the enhanced system shows less and less concepts while the original system's number of concepts reaches a really high peak (around $10^{5}$ ) and then quickly diminishes.

Reading Structural Lattices. We now try to understand what kind of information can be gleaned from structural lattices. We begin by observing the most salient properties of the systems, that is, those lattices obtained with the higher values of the pivot. Afterwards we try to bring more detail into the picture by decreasing the value of the pivot so as to vary the number of concepts from right to left as suggested by figure 2. We thus obtain a sequence of structural lattices starting from the least complex (and the least number of concepts) and gradually increasing the complexity as new concepts appear.

The first thing we can notice in both the sequence from the original and the enhanced system is the appearance of the silence attribute concept (tagged '-' in the figures). This is a well-known peculiarity of systems such as those we explore in this example and it is therefore a good sign that our analysis is progressing correctly.

Figure 3 shows a more advanced stage of analysis for a pivot $\varphi=-0.40$. In it some groups of Mandarin objects are assigned just to three English attribute concepts. Both silence and $r$ are attributed to several different Mandarin phonemes which can be interpreted as an error of the system. However, $u w$ is always attributed to Mandarin vowel sounds which, at this level of detail, seems to be a good choice.

As we continue our analysis we can find lattices such as those in figures 4 and 5 . We have omitted here the objects names that clutter the picture but the radius of the nodes is proportional to the number of objects pertaining just to them. Here, in contrast with figure 3 where most of the objects were assigned the top concept, they are all distributed into the non-extremal nodes. We can 


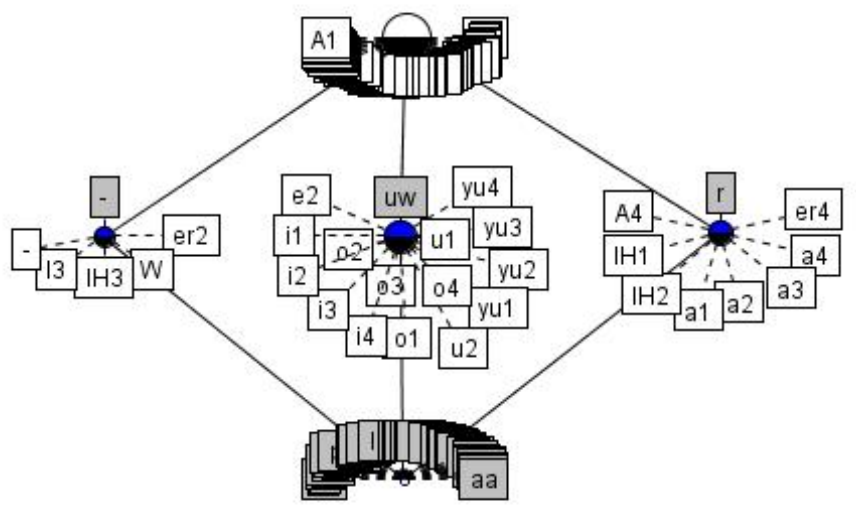

Fig. 3. Structural lattice of the enhanced system with a $\varphi=-0.40$ and 5 concepts.

also observe that these objects are grouped (most of the times) meaningfully from the point of view of their acoustic properties.

Although we show no picture here due to the large number of concepts, it is interesting to consider the lattice corresponding to the leftmost constant portion of figure 2. Despite the difficulty of drawing any conclusion from such a big lattice the most salient characteristic is that some English phonemes remain attached to the bottom concept which could be interpreted as the systems being unable to assign those English phonemes to any of the Mandarin, either due to limitations of the system or to some intrinsic characteristics of these phoneme sets.

\section{Conclusion}

We have presented an attempt at the solution of two problems of $\mathcal{K}$-Formal Concept Analysis of different ilk: first, the lack of an analogue for the second half of the basic theorem of Formal Concept Analysis, and second, the lack of a building procedure for the $\mathcal{K}$-Concept Lattice.

For the first purpose we have introduced the concept of the structural lattice of the $\mathcal{K}$-Concept Lattice based in the similar structural semilattice, as featured in idempotent algebra. Thus we expect the structural lattice to provide the "scaffolding" for the bigger $\mathcal{K}$-concept lattice. As to the relation of the structural lattice to the lattice described in the second half of the Basic Theorem of Formal Concept Analysis, we recall that (for $n, p \in \mathbb{N}$ ) Formal Concept Analysis may be taken to be the particular case of $\mathcal{K}$-Formal Concept Analysis when the idempotent reflexive semiring is the Boolean semiring, and the pivot is $\perp_{\mathbb{B} o p}=\mathbf{1}$, $\underline{\mathfrak{B}}(G, M, I)=\underline{\mathfrak{B}}^{\mathbf{1}}(G, M, I)_{\mathbb{B}}$. It is easy to see that in that case the definition 


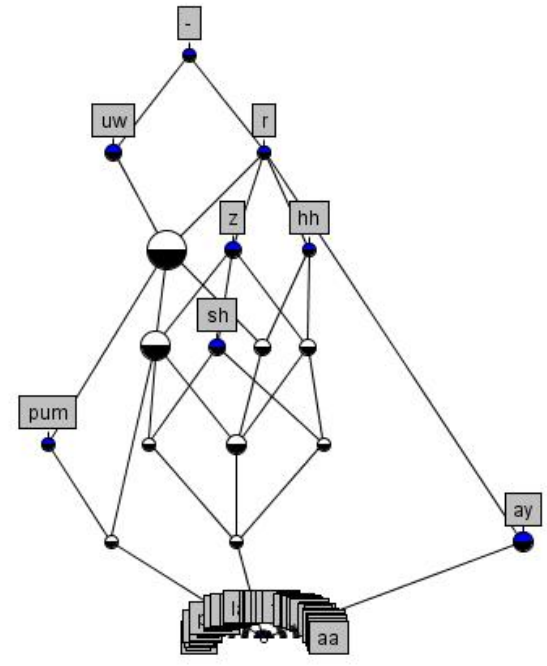

Fig. 4. Structural lattice of the enhanced system with a $\varphi=-0.99$ and 18 concepts.

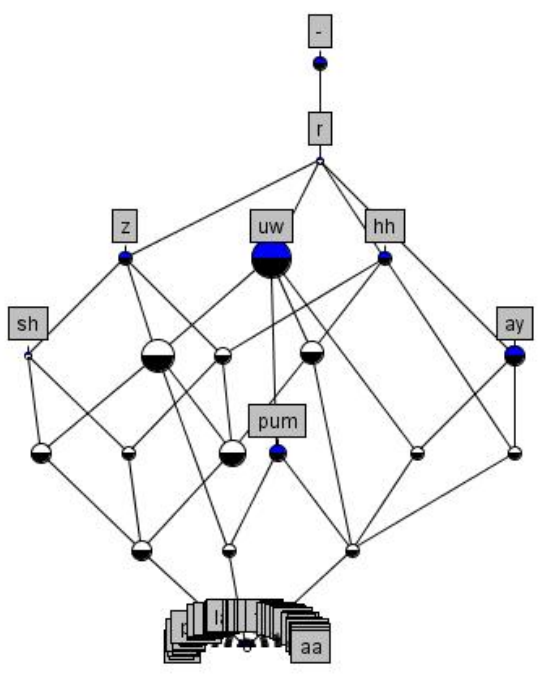

Fig. 5. Structural lattice of the enhanced system with a $\varphi=-1.09$ and 20 concepts. 
of the structural lattice and the lattice of the second part of Theorem 3 of [12] coincide.

Secondly, we have provided an algorithm to build the structural lattice by reducing its calculations to those of a standard Concept Lattice, and have used such construction to analyse the behaviour of the confusion matrices of multiple input-multiple output classifiers. We have also discussed the role of the pivot $\varphi$ introduced in [1] to modulate the Galois connection between the spaces of (multi-valued) sets of objects and attributes in such a setting and we have tried to argue how the performance of the classifier relates to its $\mathcal{K}$-Concept Lattice conforming to a particular, expected shape.

Acknowledgements We would like to thank J. Frankel for providing the classifier systems and to N. Morgan for comments on the intuitions afforded by the analysis technique.

\section{References}

1. Valverde-Albacete, F.J., Peláez-Moreno, C.: Towards a generalisatioin of formal concept analysis for data mining purposes. In Ganter, B., Kwuida, L., eds.: Proceedings of the International Conference on Formal Concept Analysis, ICFCA06. Volume 3874 of LNAI., Springer (2006) 161-176

2. Baccelli, F., Cohen, G., Olsder, G., Quadrat, J.: Synchronization and Linearity. Wiley (1992)

3. Davey, B., Priestley, H.: Introduction to lattices and order. 2nd edn. Cambridge University Press, Cambridge, UK (2002)

4. Cohen, G., Gaubert, S., Quadrat, J.P.: Duality and separation theorems in idempotent semimodules. Linear Algebra and Its Applications 379 (2004) 395-422

5. Golan, J.S.: Semirings and Their Applications. Kluwer Academic (1999)

6. Golan, J.S.: Power Algebras over Semirings. With Applications in Mathematics and Computer Science. Volume 488 of Mathematics and its applications. Kluwer Academic, Dordrecht, Boston, London (1999)

7. Gaubert, S.: Two lectures on max-plus algebra. Support de cours de la 26-iéme École de Printemps d'Informatique Théorique (1998) http://amadeus.inria.fr/gaubert/papers.html.

8. Gaubert, S., the Maxplus Group: Methods and applications of $(\max ,+)$ linear algebra. Technical Report 3088, INRIA - (1997)

9. Wagneur, E.: Moduloïds and pseudomodules 1. dimension theory. Discrete Mathematics 98 (1991) 57-73

10. Wagneur, E.: The geometry of finite dimensional pseudomodules. In: Proceedings of the 34th Conference on Decision \& Control, New Orleans, LA (1995)

11. Cunninghame-Green, R., Butković, P.: Bases in max-algebra. Linear Algebra and its Applications 389 (2004) 107-120

12. Ganter, B., Wille, R.: Formal Concept Analysis: Mathematical Foundations. Springer, Berlin, Heidelberg (1999)

13. Yevtushenko, S.A.: System of data analysis "Concept Explorer". In: Proceedings of the 7th national conference on Artificial Intelligence KII-2000, Russia, ACM (2000) 127-134 (In Russian) http://sourceforge.net/projects/conexp. 